\title{
Genetic Background Influences Brassinosteroid-Related Mutant Phenotypes in Rice
}

\author{
Tomoaki Sakamoto $^{1^{*}}$, Hidemi Kitano $^{2}$, Shozo Fujioka $^{3}$ \\ ${ }^{1}$ Faculty of Bioresources and Environmental Sciences, Ishikawa Prefectural University, Nonoichi, Japan; ${ }^{2}$ Bioscience and Biotech- \\ nology Center, Nagoya University, Nagoya, Japan; ${ }^{3}$ RIKEN Advanced Science Institute, Wako, Japan. \\ Email: "sakamoto@ishikawa-pu.ac.jp
}

Received September $30^{\text {th }}$, 2012; revised November $5^{\text {th }}$, 2012; accepted November $12^{\text {th }}, 2012$

\begin{abstract}
In two cases, mutations in the same brassinosteroid-related genes caused different phenotypes in japonica varieties Nipponbare and Taichung 65. The mutant phenotypes were less severe in the Taichung 65 background than in the Nipponbare background. Three newly isolated brassinosteroid-insensitive mutants $(d 61-1 N, d 61-11$, and $d 61-12)$ derived from a Nipponbare mutant library were found to be alleles of $d 61$, which represent defects in the OsBRII gene. Although the Nipponbare-derived mutant $d 61-1 N$ had the same nucleotide substitution as the previously characterized Taichung 65-derived mutant $d 61-1 T$, these two mutants showed different phenotypes for plant stature, internode elongation pattern, and seed shape; in each case, $d 61-1 N$ (in the Nipponbare genetic background) had the more severe mutant phenotype. Similar trends were seen for phenotypes caused by mutants of $d 2$, a brassinosteroid biosynthesis gene. Consistent with these phenotypes, the expression of brassinosteroid-responsive genes was lower in the Nipponbare-derived mutants. These results can be explained by our findings that feed-forward up-regulation of $O s B R I 1$ did not occur in the Nipponbare-derived mutants and that an mPing transposon is inserted into the promoter region of Nipponbare $O s B R I 1$. Based on these results, we conclude that the expression of $O s B R I 1$, especially its feed-forward up-regulation, is misregulated in wild-type Nipponbare and in brassinosteroid-related mutants in a Nipponbare genetic background. Although Nipponbare is a model rice genotype, it can be categorized as an OsBRI1 mutant that has reduced sensitivity to brassinosteroid.
\end{abstract}

Keywords: Brassinosteroid; Brassinosteroid Receptor; Brassinosteroid Sensitivity; miniature Ping (mPing); Mutant; Nipponbare; Rice

\section{Introduction}

Brassinosteroids are essential phytohormones for various growth and developmental processes in higher plants such as cell and stem elongation, dark-adapted morphogenesis (skotomorphogenesis), responses to environmental stress, and tracheary element differentiation [1-3]. The major pathway for brassinosteroid biosynthesis was elucidated in Arabidopsis, and a number of dwarf mutants have been identified as brassinosteroid deficient [4,5]. Brassinosteroid biosynthesis starts with the plant sterol campesterol, and the bioactive brassinosteroids, castasterone and brassinolide, are synthesized by sequential reactions catalyzed mainly by six enzymes. Among the six major enzymes involved in brassinosteroid biosynthesis in plants, four are cytochrome P450 monooxygenases. In rice, C-22 hydroxylase is encoded by CYP90B2/ OsDWARF4 and CYP724B1/D11 [6]; C-23 hydroxylase is encoded by $C Y P 90 D 2 / D 2$ and $C Y P 90 D 3$ [7]; and C-6

"Corresponding author. oxidase is encoded by CYP85A1/OsDWARF [8,9]. $C Y P 90 A s$, which are encoded by $C Y P 90 A 3 / O s C P D 1$ and CYP90A4/OsCPD2, are also believed to be involved in brassinosteroid biosynthesis in rice, although their catalytic function has not been clarified [10]. Because the most biologically active brassinosteroid, brassinolide, has been detected in Arabidopsis and some other dicot plants but not in rice, the precursor of brassinolide, castasterone, is believed to act as the bioactive brassinosteroid in rice [11]. Consistent with this hypothesis, only a single copy of the CYP85A gene is found in the rice genome, whereas duplication of this gene has occurred in most dicots and one of the genes in each pair encodes a brassinolide synthase function [12].

Genetic and molecular studies have also identified key components of the brassinosteroid signaling pathway, which include membrane receptor kinases (BRI1 and SERKs including BAK1), intracellular kinases (BIN2 and BSKs) and phosphatase (BSU1), and nuclear transcription factors (BES1 and BZR1); subsequent bio- 
chemical studies have revealed many details about signaling events from brassinosteroid perception at the cell surface to gene expression in the nucleus $[13,14]$. In rice, the putative brassinosteroid receptor gene $O s B R I 1$, and its loss-of-function mutants, $d 61$, have been identified [15]. Although the first two $d 61$ mutants identified were weak alleles, each having a single amino acid substitution, subsequent analysis added eight alleles including four null mutants having severely malformed phenotypes [16]. Two other brassinosteroid-insensitive rice mutants, dwarf and low-tillering (dlt) and leaf and tiller angle increased controller (lic), have recently been identified $[17,18]$. DLT encodes a GRAS-family transcription factor and probably acts downstream of a putative rice BZR1 ortholog. OsLIC encodes an ancestral and unique CCCH-type zinc finger protein and probably acts as an antagonistic transcription factor of a putative rice BZR1 ortholog. dlt and lic-1 showed semi-dwarf and erect-leaf phenotypes, which were also observed for the weak alleles of $d 61$, suggesting that such phenotypes are common in brassinosteroid-insensitive rice mutants.

In this study, we have characterized three rice mutants having dwarf and erect-leaf phenotypes. Because these mutants also showed a short-grain phenotype that was not observed in previously identified $d 61$ alleles, we hypothesized that these mutants have defects in novel brassinosteroid signaling components.

\section{Materials and Methods}

\subsection{Characterization of New Brassinosteroid-Related Mutants}

To obtain brassinosteroid-related mutants, we performed large-scale screening of rice mutant collections that were produced by using a retrotransposon (Tos17), chemical mutagens, and $\gamma$-ray irradiation. Three candidate lines (d61-1N, d61-11, and d61-12) were obtained from a Nipponbare library of tissue culture-induced mutations [19]. Seeds of wild-type Nipponbare and the mutants were sterilized in $1 \% \mathrm{NaClO}$ for $30 \mathrm{~min}$ and sown on Murashige and Skoog agar medium. For the initial phytohormone treatment, seedlings were grown in a growth chamber at $28^{\circ} \mathrm{C}$ under continuous light for 2 weeks, and then transplanted into medium containing $10 \mathrm{nM}$ brassinolide. For the subsequent field experiments, seedlings were grown for 1 month in a greenhouse, and then transplanted into a paddy field. For the gene expression analysis, we selected 10-day-old seedlings that exhibited uniform growth and adapted them to hydroponic culture for 2 days before treatment. Brassinolide treatment (100 $\mathrm{nM}$ ) was carried out by adding the pure chemical to the culture medium. Whole seedlings were harvested $3 \mathrm{~h}$ after the treatment and used for RNA isolation as described below.

\subsection{Mapping and Sequence Analyses}

For the mapping of each mutant, we performed linkage analysis using an $F_{2}$ population of about 2000 plants derived from crosses between the Nipponbare-derived mutant (japonica) and wild-type Kasalath (indica). The mutation sites of all three candidate lines were mapped onto the long arm of chromosome 1 , tightly linked to the $d 61$ locus (OsBRI1 gene). The nucleotide sequence of the OsBRI1 gene (from positions -4825 to +4571 , taking the translation initiation site as +1 ) from these three lines, Nipponbare, and Taichung 65 was determined by using a dideoxynucleotide chain-termination method using an automated sequencing system (ABI377; Applied Biosystems, Foster City, CA, USA), and was analyzed by using the Lasergene software (DNAStar, Inc., Madison, WI, USA).

\subsection{Plasmid Constructs and Plant Transformation}

For complementation of the $d 61-1 N$ mutant, we amplified the wildtype (Nipponbare) genomic sequence of $D 61$ (OsBRI1) from -4825 to +4571 (taking the translation initiation site as +1 ) by PCR and cloned into pBluescript II SK (Stratagene, La Jolla, CA, USA). The sequence of the amplified fragment was determined as described above, and the fragment was then cloned into the hygromycin-resistant binary vector pCAMBIA 1300 . The resulting construct was introduced into Agrobacterium tumefaciens strain EHA105, and Agrobacterium-mediated transformation of the $d 61-1 N$ mutant was performed as described [20]. Transgenic plants were selected on medium containing $50 \mathrm{mg} \cdot \mathrm{L}^{-1}$ hygromycin.

\subsection{Gene Expression Analysis}

Total RNA was extracted from whole seedlings by using an RNeasy Plant Mini Kit (Qiagen, Valencia, CA, USA). Single-strand cDNAs were synthesized by using the Advantage RT-for-PCR Kit (Clontech, Palo Alto, CA, USA). Quantitative RT-PCR was performed with an iCycler iQ real-time PCR system (Bio-Rad Laboratories, Hercules, CA, USA). The primer sequences were 5'-GTAGCCAG CTTGATCTCATCTC-3' and 5'-GGGACGACTCTAC TGCATCA-3' for BU1, 5'-TGATCCATTCCTGTACC CTG-3' and 5'-TACCTTCTTCCTCCCATCTG-3' for CYP85A1, 5'-CAGCTACTTGGCTATCTTGAAGCTC AGC-3' and 5'-CCATTCTTGTTGAAGGTGTACTCC GTGC-3' for OsBRI1, and 5'-CGCCAGTTTGGTC GCTCTCGATTTCG-3' and 5'-TCAGGAGCTCCGTG CTCTTCTGGTAC-3' for Histone H3. These primers specifically amplified the target gene sequences. Expression levels were normalized against the values obtained for Histone H3, which was used as an internal reference gene. 


\section{Results and Discussion}

\subsection{Isolation of Three Mutants with Brassinosteroid-Insensitive Phenotypes}

As the result of a large-scale screening of rice mutant collections, we identified three lines that showed the morphological characteristics of brassinosteroid-related mutants, namely dwarf plant stature and erect leaves. These mutants were obtained from a Nipponbare library of tissue culture-induced mutations [19]. The most wellknown physiological effect of brassinosteroids on growth and development of rice is the increasing of leaf angle (leaf lamina inclination), which has been used as a sensitive bioassay for brassinosteroids [21-24]. When wildtype seedlings were treated with brassinolide, the leaf angles increased (Figure 1(a)). However, exogenously applied brassinolide did not affect the leaf angle of the mutants (Figures 1(b)-(d)), suggesting that these mutants are brassinosteroid insensitive. Previously isolated brassinosteroid-insensitive rice mutants have been categorized into two groups on the basis of leaf morphology and gross morphology. Plants exhibiting a group-1 phenotype formed only abnormal leaves with stiff, tortuous blades, and their leaf sheaths were scarcely developed. These plants did not flower, exhibit internode elongation, or bear seeds. These phenotypes were observed in $d 61-3$, $d 61-4, d 61-5$, and $d 61-6$ [16]. Plants exhibiting a group-2 phenotype showed a range of semi-dwarf phenotypes (about 70\% - 95\% height of wild-type). The leaves of these plants were erect, but the other abnormal phenotypes such as twisted leaf blades were not observed. Reproductive development of group-2 phenotype plants seems normal. These phenotypes were observed in

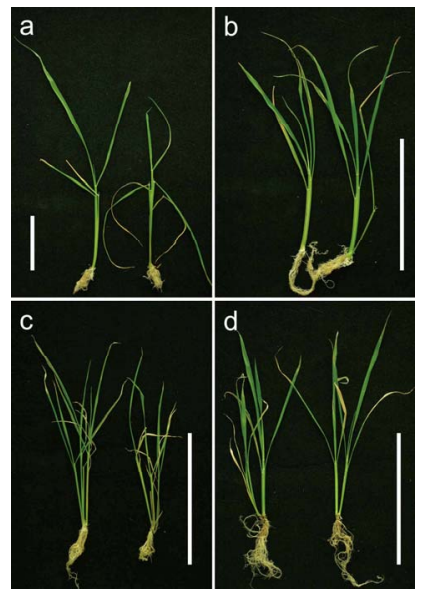

Figure 1. Effect of exogenous brassinolide treatment on the leaf angle of wild-type and mutant seedlings. (a) Wild-type Nipponbare; (b) d61-1 $N$; (c) d61-11; (d) d61-12. Each panel shows 2-week-old seedlings transplanted into MS medium containing either $10 \mathrm{nM}$ brassinolide dissolved in ethanol (right) or ethanol alone (left) and grown for another 2 weeks. Bars, $10 \mathrm{~cm}$. $d 61-1, d 61-2, d 61-7, d 61-8, d 61-9, d l t$, and lic-1 [15, $17,18,25]$. Because the gross morphology of newly isolated three mutants were ranged between group- 1 and group-2 (discuss below), we hypothesized that these mutants had defects in one or more novel brassinosteroid signaling components or had novel defects in one or more previously identified brassinosteroid signaling components.

\subsection{The Three New Brassinosteroid-Insensitive Mutants Were New d61 Alleles}

For the mapping of each mutant, linkage analysis was performed using an $F_{2}$ population of $\sim 2000$ plants derived from crosses between the mutant (japonica) and Kasalath (indica) varieties. The mutation sites of all three mutant lines were mapped onto the long arm of chromosome 1 , tightly linked to the $d 61$ locus (OsBRI1 gene). OsBRI1 encodes the rice ortholog of Arabidopsis BRI1, a leucine-rich-repeat receptor-like kinase that functions as a brassinosteroid receptor [15]. Sequence analysis of OsBRI1 revealed that one of the three lines had a single nucleotide substitution (ACC to ATC) that resulted in a substitution of amino acid residue Thr-988 (in the kinase domain) with Ile. Because the same nucleotide substitution was found in the previously characterized $d 61-1$ mutant, in this paper, we designated this new mutant allele as $d 61-1 N$ (d61-1 from wild-type Nipponbare) and renamed $d 61-1$ as $d 61-1 T$ (d61-1 from wild-type Taichung 65$)$. The other two lines (d61-11 and $d 61-12)$ had single nucleotide substitutions (GGG to GAG and GGG to AGG) that resulted in substitution of amino acid residue Gly-643 in the leucine-rich repeats with Glu and Arg, respectively (Figure 2(a)). The mutant phenotype of $d 61-1 N$ was complemented by the introduction of a 9.4$\mathrm{kb}$ genomic segment containing the OsBRI1 gene (from positions -4825 to +4571 , taking the translation initiation site as +1 ; Figure 2(b)). Based on these results, we conclude that abnormal phenotype of $d 61-1 N$ is caused by a defect in the OsBRI1 gene, and considered that all three lines identified in this study contain novel $d 61$ mutant alleles.

\subsection{Phenotypic Variation in the d61 Mutants}

The previously identified brassinosteroid-insensitive $d 61$ alleles could be categorized into two groups (weak and strong alleles) by their leaf and gross morphology [16,25]. The culm length of Taichung 65, the strain from which $d 61-1 T$ was derived (hereafter, "original strain”), was about $110 \mathrm{~cm}$, whereas that of weak allele $d 61-1 T$ was about $75 \mathrm{~cm}$ (Figures 3(a) and (b)). In wild-type rice, the leaf blade bends away from the vertical axis of the leaf sheath toward the abaxial side, whereas almost all of the leaves of $d 61-1 T$ were erect (Figure 3(c))). The other 
weak $d 61$ alleles showed similar semi-dwarf phenotypes, with plant heights ranging between 60 and $100 \mathrm{~cm}$ [25]. On the other hand, strong alleles such as $d 61-4$ formed only abnormal leaves with twisted, stiff blades, and the leaf sheath was scarcely developed (Figure 3(d)). These plants did not flower, show internode elongation, or bear any seeds, as described previously [16].

The three newly obtained lines had similar phenotypes, including severely dwarfed stature and completely erect leaves. The culm length of Nipponbare, the original strain of $d 61-1 N$, was about $90 \mathrm{~cm}$, whereas those of $d 61-1 N, d 61-11$, and $d 61-12$ were about $25 \mathrm{~cm}$ (Figures 3(e)-(h)). Although the ratio of leaf sheath length to leaf blade length was reduced in the mutants, other abnormal leaf morphologies such as the twisted leaf blade observed in the $d 61$ strong alleles (such as $d 61-4$; Figure 3(d)) were not observed. In addition to the mutant phenotypes

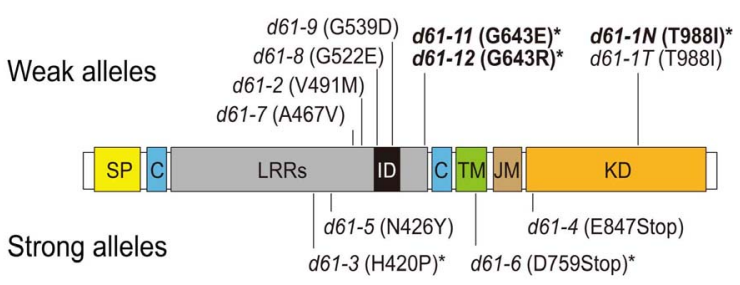

(a)

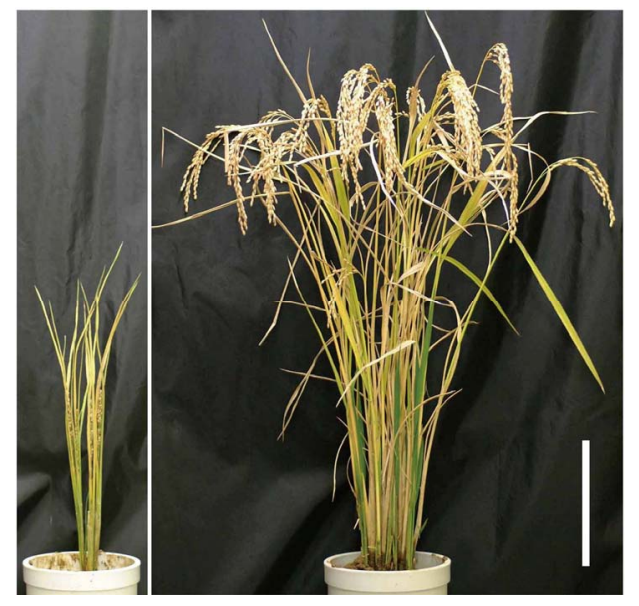

(b)

Figure 2. Characterization of the $d 61$ mutants. (a) Modular structure of the OsBRI1 (D61) protein and positions of the mutations in the three $d 61$ alleles discovered in the present study (d61-1 $N$, d61-11, and d61-12; bold type) and the previously identified alleles (d61-1 to d61-9). SP, C, LRRs, ID, TM, JM, and KD indicate signal peptide, cysteine pair, leucine-rich repeats, island domain, transmembrane domain, juxtamembrane domain, and kinase domain, respectively. Asterisks indicate alleles derived from the wild-type, Nipponbare. (b) Complementation of the abnormal morphology of the d61-1N mutant by introduction of the OsBRI1 gene. Panels show the d61-1N mutant containing the empty vector (left) and containing the DNA fragment encompassing the entire OsBRI1 gene (right). Bar, $20 \mathrm{~cm}$. of the vegetative organs of the Nipponbare-derived $d 61$ mutants, abnormal morphology was also found in the grains. Although the grains of $d 61-1 T$ were indistinguishable from those of its original strain, Taichung 65 (Figures 3(i) and (j)), those of $d 61-1 N, d 61-11$, and $d 61-12$ were visibly shorter and smaller than those of their original strain, Nipponbare (Figures 3(k)-(n)).

The phenotypes of $d 61-1 N, d 61-11$, and $d 61-12$ were less severe than those of the $d 61$ strong alleles (such as $d 61-4)$ : all three lines showed culm elongation, flowering, and normal leaf blade morphology. On the other hand, the phenotypes of the three Nipponbare-derived $d 61 \mathrm{mu}-$ tants were more severe than those of the $d 61$ weak alleles (such as $d 61-1 T$ ): all three lines had severely dwarfed stature and small grains, neither of which was observed in $d 61-1 T$. Among the previously identified brassinosteroid-related rice mutants, only the brassinosteroid-deficient $d 11$ mutants showed the small-grain phenotype, although their culm length reached $60 \%$ of that of their original strains [26]. Based on these observations, we conclude that $d 61-1 N, d 61-11$, and $d 61-12$ have unique brassinosteroid-related abnormal phenotypes and hypothesize that these mutants have novel defects in the function of brassinosteroid receptor OsBRI1.

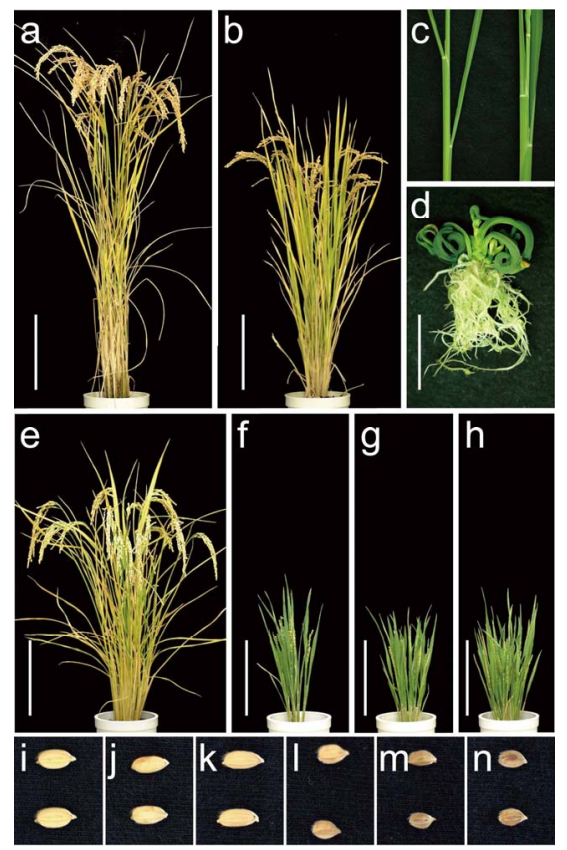

Figure 3. Phenotypic variation in the $d 61$ mutants. (a, b) Gross morphologies of wild-type Taichung 65 (a) and the Taichung 65-derived d61-1T mutant (b). (c) Comparison of the leaf angle between Taichung 65 (left) and d61-1T (right). (d) Gross morphology of a strong allele, d61-4. (e-h) Gross morphologies of wild-type Nipponbare (e) and Nipponbare-derived $d 61-1 N(f), d 61-11(g)$, and d61-12 (h) mutants. (i-n) Grain morphologies of Taichung 65 (i), d61-1T (j), Nipponbare (k), d61-1N (l), d61-11 (m), and d61-12 (n). Bars, $20 \mathrm{~cm}$ in (a, b,e-h), and $3 \mathrm{~cm}$ in (d). 


\subsection{The Same Nucleotide Substitution Caused Different Abnormal Phenotypes in Two japonica Varieties}

Next, we compared the pattern of internode elongation between $d 61-1 N$ and $d 61-1 T$ to characterize the mutant phenotypes in detail. In rice, the junction of the culm and the spike is referred to as the neck of the spike. We numbered the internodes of each culm from top to bottom; i.e., the internode just below the neck of the spike was called the first internode. Dwarf mutants of rice have been categorized into six groups based on the elongation pattern of the upper four to five internodes [27], and we attempted to place the new mutants into these categories. $d 61-1 T$ showed specific inhibition of second-internode elongation; therefore, it was grouped into the $d m$ type (Figures 4(a), (b), (f) and (g)). In contrast, the elongation of all internodes below the first internode was severely suppressed in $d 61-1 N$, which clearly fit into the $d 6$ type (Figures 4(c), (f) and (g)). $d m$-type internode elongation patterns were also observed in the brassinosteroid-deficient $d 2$ and $d 11$ mutants [26,28], suggesting that a stunted second internode is one of the characteristic phenotypes of brassinosteroid-related rice mutants. Although the reason why the elongation of second internode is specifically inhibited in these mutants has not yet been clarified, it is possible that brassinosteroids produced in the developing spike at a particular stage are necessary for second-internode elongation because internode differentiation occurs successively from top (first internode) to bottom, and synchronously with spike development. On the other hand, the second through fourth internodes were stunted in the $d 6$-type mutant $d 61-1 N$, indicating that incomplete internode differentiation occurred for a prolonged period during culm development.

In many japonica varieties, the basal rachis internode of the spike (just above the neck) scarcely elongates; however, it was elongated in both $d 61-1 N$ and $d 61-1 T$ (Figures 4(a) and (c)-(g)). This is very interesting because it indicates that partial suppression of brassinosteroid receptor function caused two inverse phenomena: reduced culm internode elongation and increased rachis internode elongation. Internode elongation depends on the intercalary meristem, whereas elongation of the rachis internode depends on the rib meristem of the shoot apical meristem [29], suggesting that brassinosteroids have opposite effects on the activity of these two meristems.

Note that the mutation in $d 61-1 N$ was identical to that in $d 61-1 T$, although the phenotypes of $d 61-1 N$ and $d 61-1 T$ were quite different (Figure 4). Because $d 61-1 N$ and $d 61-1 T$ were derived from two different japonica varieties (Nipponbare and Taichung 65, respectively), our results suggest that the genetic background of a mutant affects the severity of the mutant phenotype. Interestingly, all of the previously identified $d 61$ weak alleles
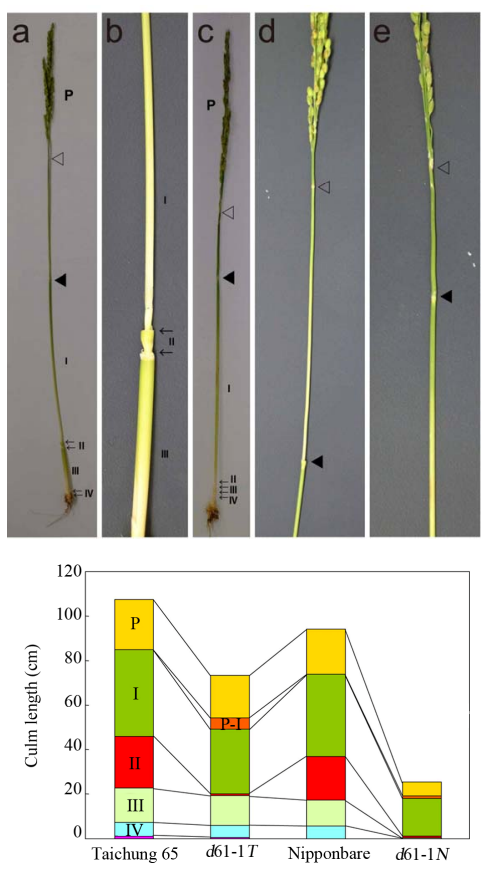

(f)

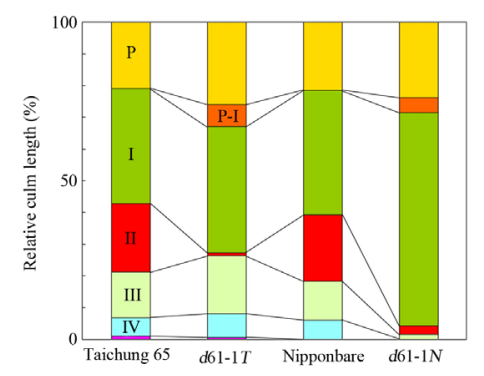

(g)

Figure 4. Comparison of the internode elongation patterns of d61-1 $N$ and d61-1T. (a-e) Elongation of internodes in $d 61-1 N$ and d61-1T. Main culm of d61-1T (a) and d61-1 $N$ (c), and close-up view of the second internode of $d 61-1 T$ (b). Close-up view of basal rachis internode of spike of d61-1T (d) and $d 61-1 N(e)$; Small arrows, closed arrowheads, and open arrowheads indicate nodes, necks, and basal rachises, respectively. Roman numerals indicate the internode number. $P$, panicle; (f) Comparison of the length of panicles and upper four internodes of Taichung 65, d61-1T, Nipponbare, and $d 61-1 N$. The lengths of panicles and upper four internodes of five main culms were averaged for each genotype. P-I, basal rachis internode of the spike; (g) Comparison of the relative lengths of panicles and upper four internodes of Taichung 65, d61-1T, Nipponbare, and d61-1N, calculated from data shown in (f).

(d61-1, d61-2, d61-7, d61-8, and d61-9) were obtained from a Taichung 65 mutant library, whereas the strong alleles include both Nipponbare-derived ( $d 61-3$ and $d 61$ 6 ) and Taichung 65-derived mutants (d61-4 and $d 61-5$; Figure 2(a)). These results indicate that severe defects in brassinosteroid receptor function induce the same abnormal phenotypes (group-1 phenotype described above) 
in mutants regardless of their genetic background. On the other hand, partial suppression of brassinosteroid receptor function resulted in different phenotypes in two different japonica varieties: the $d 61-1$ mutant phenotype was less severe in the Taichung 65 background than in the Nipponbare background.

\subsection{Different Background-Dependent Abnormal Phenotypes Were also Observed in Brassinosteroid-Deficient Mutants}

We want to point out that differences in phenotype were also observed for Nipponbare- and Taichung 65-derived brassinosteroid-deficient mutants. ebisu dwarf (d2) mutants are caused by defects in the rice brassinosteroid biosynthetic enzyme gene CYP90D2/D2 [28]. CYP90D2/ $D 2$ encodes a cytochrome P450 monooxygenase that functions in brassinosteroid C-23 hydroxylase [7]. We have isolated three new $d 2$ alleles derived from a Nipponbare mutant library $(d 2-3, d 2-4$, and $d 2-6)$, and showed that they produced more severe dwarf phenotypes in the Nipponbare genetic background than did $d 2-1$, a previously characterized null allele from a Taichung 65 mutant library [28], produced in the Taichung 65 genetic background [30].

The phenotype of Taichung 65-derived null allele $d 2-1$ was semi-dwarf: plants reached $\sim 55 \%$ (about $60 \mathrm{~cm}$ ) of the total culm length of wild-type Taichung 65 (about $110 \mathrm{~cm}$; Figures 5(a), (b) and (e)). Interestingly, the total culm length of the Nipponbare-derived null allele $d 2-6$ (about $15 \mathrm{~cm}$ ) was only about $16 \%$ of that of wild-type Nipponbare (about $95 \mathrm{~cm}$; Figures 5(c), (d) and (e)). We also compared the pattern of internode elongation between $d 2-6$ and $d 2-1$. As was the case with $d 61$, a stunted second internode ( $d m$-type pattern) and elongated basal rachis internode were observed in the Taichung 65-derived allele $d 2-1$, whereas the elongation of internodes II and below was severely suppressed in the Nipponbarederived allele $d$ 2-6 (d6-type pattern; Figures 5(e) and (f)). These results indicate that in two cases ( $d 2$ and $d 61-1)$, the severity of phenotype conferred by mutants at the same locus differed between the two genotypes, whereas the phenotypes of mutants at two different loci were similar when evaluated within the same genetic background. In other words, the dwarf phenotypes conferred by $d 61$ weak alleles and $d 2$ null alleles are strongly affected by the genetic background in which they are evaluated.

\subsection{Genetic Background Influenced the Expression of Brassinosteroid-Related Genes}

To compare the brassinosteroid response between the Nipponbare- and Taichung 65-derived mutants, we monitored the effect of brassinolide treatment on the expres- sion of $B U 1$ and $C Y P 85 A 1$. BU1 encodes a helix-loophelix protein that participates in the regulation of rice lamina inclination [31]. $B U 1$ is thought to be a primary brassinosteroid-responsive gene, because brassinosteroids increase $B U 1$ expression even in the presence of the protein synthesis inhibitor cycloheximide. CYP85A1 encodes a cytochrome P450 monooxygenase that functions in brassinosteroid C-6 oxidation, and its expression is regulated by a homeostatic system that controls bioactive brassinosteroid levels [8].

The level of BU1 mRNA in Taichung 65 seedlings was increased by brassinolide treatment to 2.4 times that in untreated plants (Figure 6(a)). Similarly, $B U 1$ expression was increased by brassinolide treatment in the
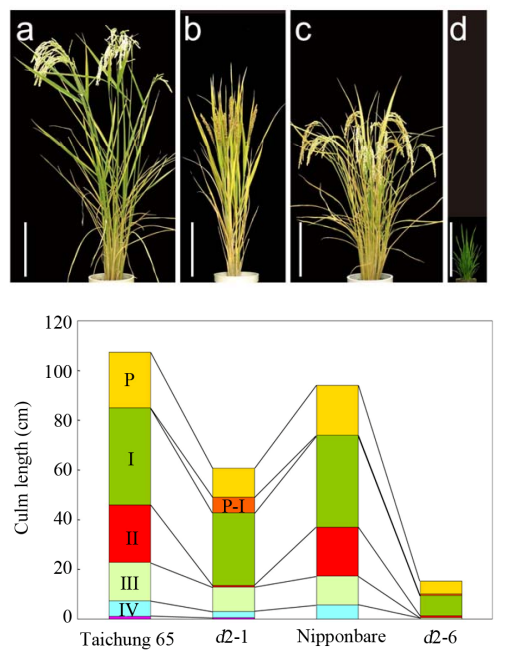

(e)

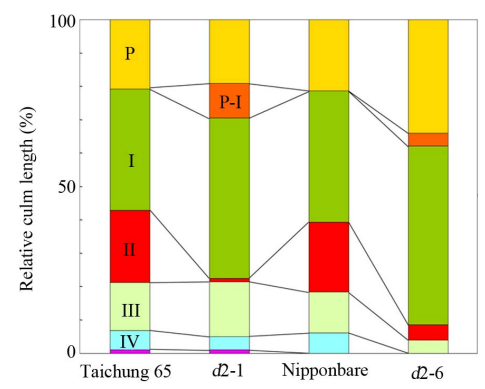

(f)

Figure 5. Phenotypic variation in the $d 2$ mutants. (a, b) Gross morphologies of wild-type Taichung 65 (a) and the Taichung 65-derived mutant d2-1 (b). (c,d) Gross morphologies of wild-type Nipponbare (c) and the Nipponbare-derived mutant d2-6 (d). Bars, $20 \mathrm{~cm}$. (e) Comparison of the length of panicles and upper four internodes of Taichung 65, d2-1, Nipponbare, and d2-6. The lengths of panicles and upper four internodes of five main culms were averaged for each genotype. Roman numerals indicate the internode number. $P$, panicle. P-I, basal rachis internode of the spike. (f) Comparison of the relative lengths of panicles and upper four internodes of Taichung 65, d2-1, Nipponbare, and d2-6, calculated from data shown in (e). 
Taichung 65-derived $d 2-1$ mutant, to 3.1 times that in untreated mutant plants. The level in the treated $d 2-1$ mutant plants was the same as that in treated wild-type Taichung 65, although the steady-state level of $B U 1$ mRNA in the absence of brassinolide treatment was lower in $d 2-1$ than in Taichung 65 (about $78 \%$ of that in Taichung 65; Figure 6(a)). This expression pattern of $B U 1$ in $d 2-1$ plants can be explained by brassinosteroid deficiency. In $d 61-1 T$, however, $B U 1$ expression was only about $74 \%$ of that in untreated Taichung 65 , regardless of brassinolide treatment (Figure 6(a)).

Expression of $B U 1$ in the Nipponbare seedlings was also increased by brassinolide treatment both in the wild-type (to 2.0 times that in untreated plants) and in the Nipponbare-derived $d 2-6$ mutant (to 2.6 times that in untreated mutant plants; Figure 6(a)), although the steady-state level of $B U 1$ mRNA in the absence of brassinolide treatment was lower in $d 2-6$ than in Nipponbare (about $72 \%$ of that in Nipponbare; Figure 6(a)). In $d 61-1 N, B U 1$ expression was about $68 \%$ of that in
Nipponbare in the absence of exogenous brassinolide and was not affected by brassinolide treatment (Figure 6(a)).

The relative increase in $B U 1$ expression caused by brassinolide treatment was less in Nipponbare (to 2.0 times) and the Nipponbare-derived $d 2-6$ mutant (to 2.6 times) than in Taichung 65 (to 2.4 times) and the Taichung 65-derived $d 2-1$ mutant (to 3.1 times). In addition, the steady-state level of $B U 1$ mRNA in the absence of brassinolide treatment was lower in $d 61-1 N$ (68\% of that in Nipponbare) than in $d 61-1 T$ (74\% of that in untreated Taichung 65). These results support our hypothesis that the response to brassinosteroid is weaker in the Nipponbare background.

The level of $C Y P 85 A 1$ mRNA in brassinolide-treated Taichung 65 seedlings decreased to about $35 \%$ of the untreated control level owing to feedback down-regulation by the homeostatic system (Figure 6(b)). Similarly, CYP85A1 expression decreased in brassinolide-treated $d 2-1$ plants (to $43 \%$ of that in untreated mutant plants), although the steady-state level of $C Y P 85 A 1$ mRNA in the (a)

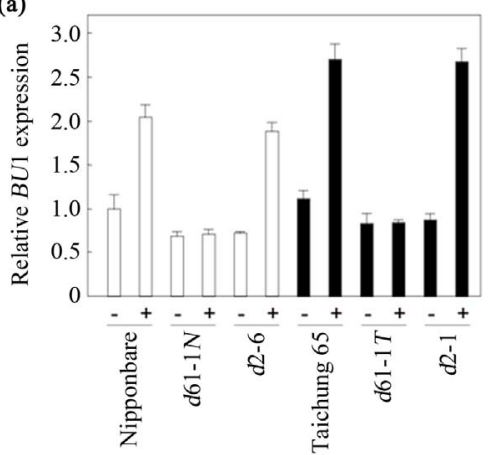

(c)

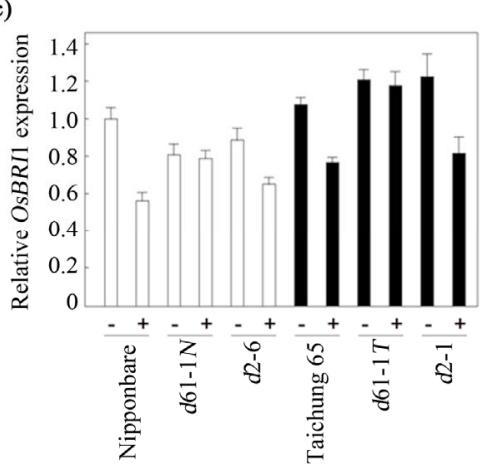

(b)

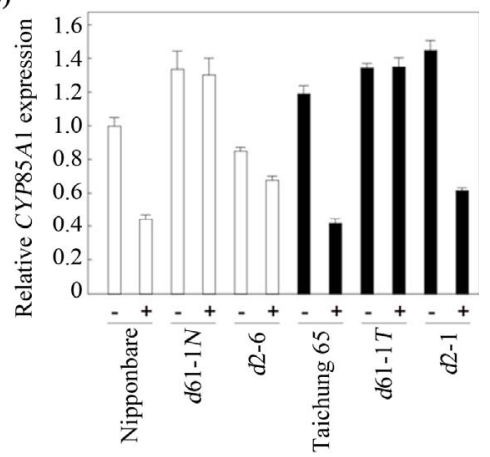

(d)

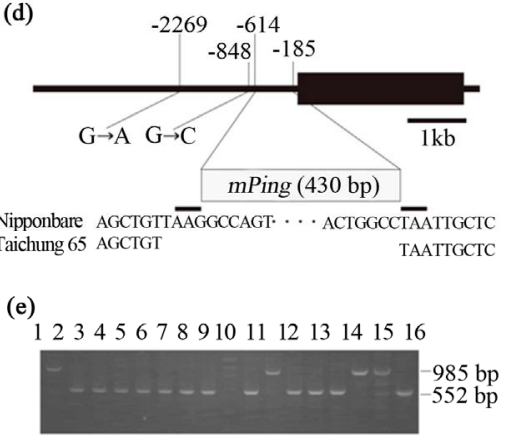

Figure 6. Expression of brassinosteroid-related genes in $d 61$ and $d 2$ mutants. (a-c) Expression of $B U 1$ (a), CYP85A1 (b), and OsBRI1 (c) in wild-type, d61-1 mutants, and $d 2$ mutants. We treated 10-day-old seedlings with $100 \mathrm{nM}$ brassinolide dissolved in ethanol (+) or ethanol alone (-) for $3 \mathrm{~h}$. Expression levels were normalized against the values obtained for $H i s t o n e ~ H 3$. The value obtained from the ethanol-treated (control) Nipponbare seedlings was then normalized to a value of 1.0. Nipponbare is the wild-type for $d 61-1 N$ and $d 2-6$; Taichung 65 is the wild-type for $d 61-1 T$ and $d 2-1$. Each bar represents the mean \pm s.d. of three biological repeats. (d) Genomic structure of OsBRI1 and the position of the $m$ Ping insertion in Nipponbare. Black box represents the single exon. Nucleotide substitutions are shown with the Nipponbare sequence given first. (e) Detection of the mPing insertion in various rice cultivars. 1, Nipponbare; 2, Taichung 65; 3, Koshihikari; 4, Sasanishiki; 5, Akitakomachi; 6, Hitomebore; 7, Haenuki; 8, Aichinokaori; 9, Norin 22; 10, Asahi; 11, IR20; 12, IR24; 13, IR26; 14, Aikawa 1; 15, Blue Rose; 16, Lemont. M, size marker ( $\varphi$ X/HaeIII). PCR fragments with and without $m$ Ping insertion are 985 bp and 552 bp, respectively. 
absence of brassinolide treatment was higher in $d 2-1$ than in wild-type Taichung 65 owing to feed-forward up-regulation by the homeostatic system (about 1.2 times that in Taichung 65; Figure 6(b)). This expression pattern of CYP85A1 in $d 2-1$ plants can also be explained by brassinosteroid deficiency. In $d 61-1 T, C Y P 85 A 1$ expression was slightly higher than wild-type in the absence of brassinolide treatment (1.1 times that in Taichung 65) and unaffected by brassinolide treatment (Figure 6(b)).

Expression of CYP85A1 in brassinolide-treated Nipponbare seedlings decreased to about $44 \%$ of the untreated control level (Figure 6(b)). Interestingly, the level of $C Y P 85 A 1$ mRNA in untreated $d 2-6$ was lower than that of Nipponbare (about 85\%), and was further decreased by brassinolide treatment (Figure 6(b)). Because the bioactive brassinosteroid castasterone accumulated in $d 2-6$ mutant [30], we hypothesize that the expression of CYP85A1 may also be regulated directly by its catalytic product, castasterone. On the other hand, the level of CYP85A1 mRNA in $d 61-1 N$ was 1.3 times that of wild-type Nipponbare and was not affected by brassinolide treatment (Figure 6(b)).

The differences in expression between the wild-type and $d 61-1$ mutant in each genotype indicate that the effect of feed-forward up-regulation on $C Y P 85 A 1$ expression was greater in $d 61-1 N$ (Nipponbare background) than in $d 61-1 T$ (Taichung 65 background), even though both $d 61-1 N$ and $d 61-1 T$ harbor the same amino acid change in the brassinosteroid receptor OsBRI1.

\subsection{Nipponbare Has Mutations in the Promoter Region of OsBRI1}

The expression analysis of $B U 1$ and $C Y P 85 A 1$ supports our hypothesis that the response to brassinosteroid is weaker in the Nipponbare background than in the Taichung 65 background. Next, we monitored the effect of brassinolide treatment on the expression of OSBRI1 in the Nipponbare- and Taichung 65-derived mutants. The level of OsBRI1 mRNA in Taichung 65 seedlings was decreased by brassinolide treatment to about $70 \%$ of that in untreated plants (Figure 6(c)). Similarly, OsBRI1 expression was decreased by brassinolide treatment in the Taichung 65-derived $d 2-1$ mutant to about $67 \%$ of that in untreated mutant plants, although the steady-state level of $O s B R I 1$ mRNA in the absence of brassinolide treatment in $d 2-1$ was 1.1 times that in Taichung 65 (Figure 6(c)). This expression pattern of $O s B R I 1$ in $d 2-1$ plants can be explained by the regulation of feedback mechanism caused by brassinosteroid deficiency. In $d 61-1 T$, OsBRI1 expression was about 1.1 times that in Taichung 65 and was not affected by brassinolide treatment (Figure 6(c)).

Expression of OsBRI1 in the Nipponbare seedlings was decreased by brassinolide treatment to about 56\% that in untreated plants (Figure 6(c)). Interestingly, however, expression of $O s B R I 1$ in the untreated $d 61-1 N$ and $d 2-6$ mutants was about $80 \%$ and $89 \%$ of that in untreated Nipponbare, respectively (Figure 6(c)). These results are clearly opposite those observed for the Taichung 65-derived $d 61-1 T$ and $d 2-1$ mutants, which had higher levels of $O S B R I 1$ than wild-type Taichung 65, and indicate that feed-forward up-regulation of OsBRI1 did not occur in the Nipponbare-derived $d 61-1 N$ and $d 2-6 \mathrm{mu}-$ tants. Feed-forward up-regulation of OsBRI1 (and the resulting increase in the amount of OsBRI1 receptor) is considered to partially compensate for the deficiency of brassinosteroids in biosynthetic mutants and for the insensitivity to brassinosteroids in receptor mutants, even though the function of OsBRI1 is partially suppressed in these mutants. Thus, a defect in regulation of OsBRI1 would explain why the response to brassinosteroid is weaker in the Nipponbare background and why the Nipponbare-derived $d 61-1 N$ and $d 2-6$ mutants showed more severe phenotypes than the Taichung 65-derived $d 61-1 T$ and $d 2-1$ mutants.

Because the expression of $O s B R I 1$ was regulated differently in the Nipponbare and Taichung 65 genetic backgrounds, we hypothesized that Nipponbare has a mutant $O s B R I 1$ gene and that the expression of OsBRI1 is misregulated in the Nipponbare genetic background. To test this hypothesis, we compared the nucleotide sequence of a 9.4-kb genomic segment containing the OsBRI1 gene (from positions -4825 to +4571 , taking the translation initiation site as +1) from Nipponbare and Taichung 65 . We found two nucleotide substitutions at positions -2269 (G to A) and -848 (G to C), and an insertion of a Tourist-like miniature inverted-repeat transposable element (MITE) of $430 \mathrm{bp,} \mathrm{miniature} \mathrm{Ping} \mathrm{(mPing)} \mathrm{[32-34],} \mathrm{in}$ the promoter region of $O s B R I 1$ from the Nipponbare genome (Figure 6(d)). The insertion was flanked on both ends by a three-nucleotide sequence, TAA, which is the typical target sequence for duplication upon $m$ Ping transposition. These structural features indicate that mPing transposed into the promoter region of OsBRI1 in Nipponbare. Ohmori et al. [35] reported that an insertion of Ping (an autonomous transposon that can activate $m$ Ping transposition) into the fourth intron of the DROOPING $L E A F(D L)$ gene decreased the transcript level of $D L$. In our case, the Nipponbare $O S B R I 1$ promoter contains an $m$ Ping insertion, and the expression of OsBRI1 in Nipponbare seems to be misregulated by the homeostatic system that controls the levels of brassinosteroid contents and signals. We also compared the nucleotide sequence of the CYP90D2 (D2) and CYP85A1 genes from Nipponbare and Taichung 65, and found no nucleotide substitution between these two cultivars. Based on these results, we conclude that the severity of the mutant phenotypes caused by the mutations in brassinosteroid-related 
genes can be explained by the insertion of $m$ Ping into the promoter of the Nipponbare $O s B R I 1$ gene.

Nipponbare is a model rice genotype being sequenced by the International Rice Genome Sequencing Project Consortium; however, based on the results here, it can be categorized as an $O s B R I 1$ mutant having reduced sensitivity to brassinosteroid. The insertion of mPing in the promoter region of OsBRI1 can be detected by PCR, and we found this insertion in several other rice cultivars we tested (Figure 6(e)), indicating that this mutation might be present in other rice cultivars investigated for brassinosteroid function. Although brassinosteroid-related mutants have not been isolated from any of these mPinginserted cultivars other than Nipponbare, we speculate that the expression of OsBRI1 is misregulated in these cultivars. These findings indicate that possible changes in sensitivity to brassinosteroids caused by this mPing insertion must be considered when the effects of brassinosteroids on the regulation of various growth and developmental processes in rice are examined.

\section{Conclusion}

In this study, we characterized three new alleles of the rice $d 61$ mutation. Although the Nipponbare-derived $d 61$ $1 N$ mutant had the same nucleotide substitution as the previously characterized Taichung 65-derived $d 61-1 T$ mutant, these two mutants showed different phenotypes for dwarfism, internode elongation pattern, and seed shape. Consistent with these phenotypes, the expression of brassinosteroid-responsive genes is lower in the Nipponbare background than in the Taichung 65 background. Because the feed-forward up-regulation of OSBRI1 seen in the Taichung 65-derived mutants was not observed in the Nipponbare-derived mutants and an mPing transposon was found in the promoter region of Nipponbare $O s B R I 1$, we conclude that the expression of OsBRI1, especially its feed-forward up-regulation, is misregulated in wild-type Nipponbare and in brassinosteroid-related mutants in a Nipponbare genetic background.

\section{Acknowledgements}

We thank Asako Tokida-Segawa for technical assistance. TS was supported by Grants-in-Aid for Young Scientists (Nos. 19688001 and 24780005) from the Ministry of Education, Culture, Sports, Science and Technology (MEXT) of Japan. SF was supported by Grants-in-Aid for Scientific Research (B) (Nos. 19380069 and 23380066) from MEXT.

\section{REFERENCES}

[1] S. D. Clouse and J. M. Sasse, "Brassinosteroids: Essential Regulators of Plant Growth and Development," Annual Review of Plant Physiology and Plant Molecular Biology,
Vol. 49, 1998, pp. 427-451. doi:10.1146/annurev.arplant.49.1.427

[2] J. M. Sasse, "Physiological Action of Brassinosteroids: An Update,” Journal of Plant Growth Regulation, Vol. 22, No. 4, 2003, pp. 276-288. doi:10.1007/s00344-003-0062-3

[3] P. Krishna, "Brassinosteroid-Mediated Stress Responses," Journal of Plant Growth Regulation, Vol. 22, No. 4, 2003, pp. 289-297. doi:10.1007/s00344-003-0058-Z

[4] G. J. Bishop and T. Yokota, "Plant Steroid Hormones, Brassinosteroids: Current Highlights of Molecular Aspects on Their Synthesis/Metabolism, Transport, Perception and Response," Plant and Cell Physiology, Vol. 42, No. 2, 2001, pp. 114-120. doi:10.1093/pcp/pce018

[5] S. Fujioka and T. Yokota, "Biosynthesis and Metabolism of Brassinosteroids," Annual Review of Plant Biology, Vol. 54, 2003, pp. 137-164.

doi:10.1146/annurev.arplant.54.031902.134921

[6] T. Sakamoto, Y. Morinaka, T. Ohnishi, H. Sunohara, S. Fujioka, M. Ueguchi-Tanaka, M. Mizutani, K. Sakata, S. Takatsuto, S. Yoshida, H. Tanaka, H. Kitano and M. Matsuoka, "Erect Leaves Caused by Brassinosteroid Deficiency Increase Biomass Production and Grain Yield in Rice,” Nature Biotechnology, Vol. 24, No. 1, 2006, pp. 105-109. doi:10.1038/nbt1173

[7] T. Sakamoto, T. Ohnishi, S. Fujioka, B. Watanabe and M. Mizutani, "Rice CYP90D2 and CYP90D3 Catalyze C-23 Hydroxylation of Brassinosteroids in Vitro," Plant Physiology and Biochemistry, Vol. 58, 2012, pp. 220-226. doi:10.1016/j.plaphy.2012.07.011

[8] Z. Hong, M. Ueguchi-Tanaka, S. Shimizu-Sato, Y. Inukai, S. Fujioka, Y. Shimada, S. Takatsuto, M. Agetsuma, S. Yoshida, Y. Watanabe, S. Uozu, H. Kitano, M. Ashikari and M. Matsuoka, "Loss-of-Function of a Rice Brassinosteroid Biosynthetic Enzyme, C-6 Oxidase, Prevents the Organized Arrangement and Polar Elongation of Cells in the Leaves and Stem," Plant Journal, Vol. 32, No. 4, 2002, pp. 495-508.

doi:10.1046/j.1365-313X.2002.01438.X

[9] M. Mori, T. Nomura, H. Ooka, M. Ishizaka, T. Yokota, K. Sugimoto, K. Okabe, H. Kajiwara, K. Satoh, K. Yamamoto, H. Hirochika and S. Kikuchi, "Isolation and Characterization of a Rice Dwarf Mutant with a Defect in Brassinosteroid Biosynthesis," Plant Physiology, Vol. 130, No. 3, 2002, pp. 1152-1161. doi:10.1104/pp.007179

[10] T. Sakamoto and M. Matsuoka, "Characterization of Constitutive Photomorphogenesis and Dwarfism Homologs in Rice (Oryza sativa L.)," Journal of Plant Growth Regulation, Vol. 25, No. 3, 2006, pp. 245-251. doi:10.1007/s00344-006-0041-6

[11] A. Bajguz and A. Tretyn, "The Chemical Characteristic and Distribution of Brassinosteroids in Plants," Phytochemistry, Vol. 62, No. 7, 2003, pp. 1027-1046. doi:10.1016/S0031-9422(02)00656-8

[12] B. K. Kim, S. Fujioka, S. Takatsuto, M. Tsujimoto and S. Choe, "Castasterone Is a Likely End Product of Brassinosteroid Biosynthetic Pathway in Rice," Biochemical and Biophysical Research Communications, Vol. 374, No. 4, 2008, pp. 614-619. 
[13] T. W. Kim and Z. Y. Wang, "Brassinosteroid Signal Transduction from Receptor Kinases to Transcription Factors,” Annual Review of Plant Biology, Vol. 61, 2010, pp. 681-704. doi:10.1146/annurev.arplant.043008.092057

[14] S. D. Clouse, "Brassinosteroid Signal Transduction: From Receptor Kinase Activation to Transcriptional Networks Regulating Plant Development,” Plant Cell, Vol. 23, No. 4, 2011, pp. 1219-1230. doi:10.1105/tpc.111.084475

[15] C. Yamamuro, Y. Ihara, X. Wu, T. Noguchi, S. Fujioka, S. Takatsuto, M. Ashikari, H. Kitano and M. Matsuoka, "Loss of Function of a Rice Brassinosteroid Insensitive1 Homolog Prevents Internode Elongation and Bending of the Lamina Joint," Plant Cell, Vol. 12, No. 9, 2000, pp. 1591-1606.

[16] A. Nakamura, S. Fujioka, H. Sunohara, N. Kamiya, Z. Hong, Y. Inukai, K. Miura, S. Takatsuto, S. Yoshida, M. Ueguchi-Tanaka, Y. Hasegawa, H. Kitano and M. Matsuoka, "The Role of OsBRII and Its Homologous Genes, OsBRL1 and OsBRL3, in Rice," Plant Physiology, Vol. 140, No. 2, 2006, pp. 580-590. doi:10.1104/pp.105.072330

[17] H. Tong, Y. Jin, W. Liu, F. Li, J. Fang, Y. Yin, Q. Qian, L. Zhu and C. Chu, "Dwarf and Low-Tillering, a New Member of the GRAS Family, Plays Positive Roles in Brassinosteroid Signaling in Rice,” Plant Journal, Vol. 58, No. 5, 2009, pp. 803-816. doi:10.1111/j.1365-313X.2009.03825.X

[18] C. Zhang, Y. Xu, S. Guo, J. Zhu, Q. Huan, H. Liu, L. Wang, G. Luo, X. Wang and K. Chong, "Dynamics of Brassinosteroid Response Modulated by Negative Regulator LIC in Rice,” PLoS Genetics, Vol. 8, 2012, e1002686. doi:10.1371/journal.pgen.1002686

[19] H. Hirochika, K. Sugimoto, Y. Otsuki, H. Tsugawa and M. Kanda, "Retrotransposons of Rice Involved in Mutations Induced by Tissue Culture," Proceedings of the $\mathrm{Na}$ tional Academy of Sciences of the United States of America, Vo. 93, No. 15, 1996, pp. 7783-7788. doi:10.1073/pnas.93.15.7783

[20] Y. Hiei, S. Ohta, T. Komari and T. Kumashiro, "Efficient Transformation of RIce (Oryza sativa L.) Mediated by Agrobacterium and Sequence Analysis of Boundaries of the T-DNA,” Plant Journal, Vol. 6, No. 2, 1994, pp. 271282. doi:10.1046/j.1365-313X.1994.6020271.x

[21] E. Maeda, "Rate of Lamina Inclination in Excised Rice Leaves,” Physiologia Plantarum, Vol. 18, No. 3, 1965, pp. 813-827. doi:10.1111/j.1399-3054.1965.tb06940.x

[22] K. Wada, S. Marumo, N. Ikekawa, M. Morisaki and K. Mori, "Brassinolide and Homobrassinolide Promotion of Lamina Inclination of Rice Seedlings,” Plant and Cell Physiology, Vol. 22, No. 2, 1981, pp. 323-325.

[23] K. Takeno and R. P. Pharis, "Brassinosteroid-Induced Bending of the Leaf Lamina of Dwarf Rice Seedlings: An Auxin-Mediated Phenomenon,” Plant and Cell Physiology, Vol. 23, No. 7, 1982, pp. 1275-1281.

[24] S. Fujioka, T. Noguchi, S. Takatsuto and S. Yoshida, "Activity of Brassinosteroids in the Dwarf Rice Lamina Inclination Bioassay,” Phytochemistry, Vol. 49, No. 7, 1998, pp. 1841-1848.

\section{doi:10.1016/S0031-9422(98)00412-9}

[25] Y. Morinaka, T. Sakamoto, Y. Inukai, M. Agetsuma, H. Kitano, M. Ashikari and M. Matsuoka, "Morphological Alteration Caused by Brassinosteroid Insensitivity Increases the Biomass and Grain Production of Rice,” Plant Physiology, Vol. 141, No. 3, 2006, pp. 924-931. doi:10.1104/pp.106.077081

[26] S. Tanabe, M. Ashikari, S. Fujioka, S. Takatsuto, S. Yoshida, M. Yano, A. Yoshimura, H. Kitano, M. Matsuoka, Y. Fujisawa, H. Kato and Y. Iwasaki, “A Novel Cytochrome P450 Is Implicated in Brassinosteroid Biosynthesis via the Characterization of a Rice Dwarf Mutant, dwarf11, with Reduced Seed Length,” Plant Cell, Vol. 17, No. 3, 2005, pp. 776-790. doi:10.1105/tpc.104.024950

[27] K. Takeda, "Internode Elongation and Dwarfism in Some Gramineous Plants,” Gamma Field Symposium, Vol. 16, 1977, pp. 1-18.

[28] Z. Hong, M. Ueguchi-Tanaka, K. Umemura, S. Uozu, S. Fujioka, S. Takatsuto, S. Yoshida, M. Ashikari, Kitano and M. Matsuoka, "A Rice Brassinosteroid-Deficient Mutant, ebisu dwarf (d2), Is Caused by a Loss of Function of a New Member of Cytochrome P450,” Plant Cell, Vol. 15, No. 12, 2003, pp. 2900-2910. doi:10.1105/tpc.014712

[29] K. Hoshikawa, “Stem,” In: K. Hoshikawa, Ed., The Growing Rice Plant, Nobunkyo, Tokyo, 1989, pp. 123-148.

[30] T. Sakamoto, Y. Morinaka, H. Kitano and S. Fujioka, "New Alleles of Rice ebisu dwarf (d2) Mutant Show Both Brassinosteroid-Deficient and -Insensitive Phenotypes," American Journal of Plant Sciences, Vol. 3, No. 12, pp. 1699-1707.

[31] A. Tanaka, H. Nakagawa, C. Tomita, Z. Shimatani, M. Ohtake, T. Nomura, C. J. Jiang, J. G. Dubouzet, S. Kikuchi, H. Sekimoto, T. Yokota, T. Asami, T. Kamakura and M. Mori, “BRASSINOSTEROID UPREGULATED1, Encoding a Helix-Loop-Helix Protein, Is a Novel Gene Involved in Brassinosteroid Signaling and Controls Bending of the Lamina Joint in Rice,” Plant Physiology, Vol. 151, No. 2, 2009, pp. 669-680. doi:10.1104/pp.109.140806

[32] N. Jiang, Z. Bao, X. Zhang, H. Hirochika, S. R. Eddy, S. R. McCouch and S. R. Wessler, "An Active DNA Transposon Family in Rice,” Nature, Vol. 421, No. 6919, 2003, pp. 163-167. doi:10.1038/nature01214

[33] K. Kikuchi, K. Terauchi, M. Wada and H. Y. Hirano, "The Plant MITE mPing Is Mobilized in another Culture,” Nature, Vol. 421, No. 6919, 2003, pp. 167-170. doi:10.1038/nature01218

[34] T. Nakazaki, Y. Okumoto, A. Horibata, S. Yamahira, M. Teraishi, H. Nishida, H. Inoue and T. Tanisaka, "Mobilization of a Transposon in the Rice Genome,” Nature, Vol. 421, No. 6919, 2003, pp. 170-172. doi:10.1038/nature01219

[35] Y. Ohmori, M. Abiko, A. Horibata and H. Y. Hirano, “A Transposon, Ping, Is Integrated into Intron 4 of the $D R O$ OPING LEAF Gene of Rice, Weakly Reducing Its Expression and Causing a Mild Drooping Leaf Phenotype," Plant and Cell Physiology, Vol. 49, No. 8, 2008, pp. 1176-1184. doi:10.1093/pcp/pcn093 